Article

\title{
Evaluating the Potential of Ursolic Acid as Bioproduct for Cutaneous and Visceral Leishmaniasis
}

\author{
Pablo Bilbao-Ramos ${ }^{1,2}$, Dolores R. Serrano ${ }^{3,4} \mathbb{D}$, Helga Karina Ruiz Saldaña ${ }^{3}$, \\ Juan J. Torrado ${ }^{3,4}$ D , Francisco Bolás-Fernández ${ }^{1}$ and María Auxiliadora Dea-Ayuela ${ }^{1,5, *}$ \\ 1 Departament of Microbiology and Parasitology, School of Pharmacy, Universidad Complutense de Madrid, \\ Plaza Ramón y Cajal s/n, 28040 Madrid, Spain; pablobil15@yahoo.com (P.B.-R.); francisb@ucm.es (F.B.-F.) \\ 2 Laboratorio de Parasitología y Entomología INLASA, Pasaje Rafael Zubieta \#1889, \\ (Lado Estado Mayor del ejército) Zona Miraflores, La Paz, Bolivia \\ 3 Departament of Pharmaceutics and Food Technology, School of Pharmacy, University Complutense, \\ Avenida Complutense, 28040 Madrid, Spain; drserran@ucm.es (D.R.S.); helgakar@ucm.es (H.K.R.S.); \\ torrado1@ucm.es (J.J.T.) \\ 4 Institute of Industrial Pharmacy (IUFI), Plaza Ramon y Cajal, SN, 28040 Madrid, Spain \\ 5 Departament of Pharmacy, School of Health Science, Universidad CEU Cardenal Herrera, \\ C/Ramón y Cajal s/n, 46115 Alfara del Patriarca (Valencia), Spain \\ * Correspondence: mdea@uchceu.es; Tel.: +34-961-369-000; Fax: +34-961-395-272
}

Academic Editors: Manuela Pintado, Ezequiel Coscueta and María Emilia Brassesco Received: 12 February 2020; Accepted: 15 March 2020; Published: 19 March 2020

\begin{abstract}
Leishmaniasis affects around 12 million people worldwide and is estimated to cause the ninth-largest disease burden. There are three main forms of the disease, visceral (VL), cutaneous (CL), and mucocutaneous (MCL), leading to more than one million new cases every year and several thousand deaths. Current treatments based on chemically synthesized molecules are far from ideal. In this study, we have tested the in vitro and in vivo efficacy of ursolic acid (UA), a multifunctional triterpenoid with well-known antitumoral, antioxidant, and antimicrobial effects on different Leishmania strains. The in vitro antileishmanial activity against the intracellular forms was six and three-fold higher compared to extracellular forms of L. amazonensis and L. infantum, respectively. UA also showed to be a potent antileishmanial drug against both VL and CL manifestations of the disease in experimental models. UA parenterally administered at $5 \mathrm{mg} / \mathrm{kg}$ for seven days significantly reduced the parasite burden in liver and spleen not only in murine acute infection but also in a chronic-infection model against $L$. infantum. In addition, UA ointment $(0.2 \%)$ topically administered for four weeks diminished (50\%) lesion size progression in a chronic infection model of CL caused by L. amazonensis, which was much greater than the effect of UA formulated as an $\mathrm{O} / \mathrm{W}$ emulsion. UA played a key role in the immunological response modulating the Th1 response. The exposure of Leishmania-infected macrophages to UA led to a significant different production in the cytokine levels depending on the Leishmania strain causing the infection. In conclusion, UA can be a promising therapy against both CL and VL.
\end{abstract}

Keywords: ursolic acid; visceral leishmaniasis; acute-infection; chronic-infection; cutaneous leishmaniasis; cytokines

\section{Introduction}

Leishmaniasis affects around 12 million people worldwide and is estimated to cause the ninth-largest disease burden [1]. There are three main forms of the disease, visceral (VL), cutaneous (CL) and mucocutaneous (MCL) [2]. Approximately 0.7 to 1.2 million new cases of CL, 0.2 to 0.4 million new VL cases, and 20,000 to 30,000 deaths occur annually. Around 310 million people are at risk of 
infection who live in one of the 98 endemic regions, most of them being developing countries [1,2]. CL causes ulcers on exposed parts of the body leading to permanent scars and disfigurement, stigma, and disability. Nevertheless, VL affects the vital organs in the body being the most severe form, which is fatal if left untreated [2]. Over twenty Leishmania spp. are known to transmit the disease to humans. According to the species and the differences in virulence, the infections may vary from simple cutaneous lesions to mucocutaneous ulcers or visceral diseases [3,4].

The chemotherapy of leishmaniasis has been based on the use of antimonial compounds, such as sodium stibogluconate and meglumine antimoniate, which are far from being the ideal treatments as they display a variable efficacy against VL and CL, cause severe side effects and are parenterally administered [5]. Currently, liposomal amphotericin B is the choice of therapy in VL. However, its high cost hampers its use in developing countries where conventional amphotericin B is still used in spite of causing severe nephrotoxicity [6]. Pentavalent antimonials are still the first-line drug but exhibit several limitations, including severe side effects, the need for daily parenteral administration and drug resistance [7]. Other medicines utilized against leishmaniasis are pentamidine and paromomycin, characterized by low cost and variable efficacy, and miltefosine, which is the only drug approved for oral administration but has the disadvantages of being teratogenic and leading to emerging resistances [8,9]. Many factors, including lack of efficacy, severe adverse effects, long and high cost therapies, and parenteral administration of most drugs, result in poor patient compliance and, at the same time, increases the risk of emerging resistant strains. Definitively, there is a clinical need for safer, more effective, and inexpensive treatments against all the leishmaniasis forms.

Currently, major research efforts are focused on discovering new natural compounds with antileishmanial properties [10]. Many active compounds with remarkable biological activities have been isolated from different plant species, such as flavonoids [11,12], anthocyanidins [13], coumarins [14], and triterpenoids [15].

Triterpenoids are polycyclic compounds derived from the linear hydrocarbon squalene and comprise one of the most promising groups of phytochemicals due to its multifunctionality in the treatment of a broad range of diseases [16-21]. For instance, oleanolic acid administered at low doses has hepatoprotective properties, whereas high doses can result in cholestasis and hepatotoxicity [22].

Ursolic Acid (UA) is a ubiquitous triterpenoid in the plant kingdom [23]. UA is a promising anticancer drug. Phase I clinical studies have already been performed in order to evaluate its toxicity and pharmacokinetic profile in healthy volunteers and patients with advanced solid tumors [24,25]. The intravenous infusion of encapsulated UA in liposomes was well tolerated, showing manageable toxicities with a maximum tolerated-dose of $98 \mathrm{mg} / \mathrm{mL}$. Besides its antitumoral activity, UA possesses a wide variety of biological activities, such as antioxidant [26], hepatoprotective [22], hypoglycaemic [27], antibacterial [26], antiviral [28], antifungal [29] and antiprotozoal [18]. Its antiprotozoal efficacy has been tested in vitro against Trypanosoma cruzi [21], Plasmodium falciparum [22], and Leishmania spp [30].

Regarding the immunological effect of triterpenes, several studies have shown that they possess anti-inflammatory activity, mainly caused either by inhibiting enzymes involved in the production of eicosanoids, such as cyclooxygenases, or inhibiting the release of cytokines [31]. This antiinflammatory effect is crucial against several infections, such as those caused by intracellular pathogens. In Mycobacterium tuberculosis, UA has shown the ability to inhibit the expression of certain inflammatory cytokines, such as TNF- $\alpha$, IL-1 $\beta$, IL-6, and TGF- $\beta$ [32,33]. UA also has exhibited activity against other intracellular pathogens, such as Toxoplasma gondii, leading to an increased production of NO, ROS, IL-10, IL-12, granulocyte macrophage colony stimulating factor (GM-CSF) and interferon- $\beta$, while reducing the expression of IL-1 $\beta$, IL-6, TNF- $\alpha$ and TGF- $\beta$ in infected immune cells [34].

Several plant extracts from Brazil have shown in vitro activity against L. amazonensis and L. braziliensis. This activity has been attributed to the fraction of Baccharis uncinellase containing oleanolic and ursolic acids [35]. The same fraction has also exhibited in vivo efficacy in tegumentary leishmaniasis, resulting in a decreased skin parasitism and increased levels of IL-12 and IFN- $\gamma$, spreading the Th1 immune response [20]. In addition, the same plant extract was effective in vivo against 
L. infantum [36]. UA extracted from Petiveria alliaceae showed similar activity against L. amazonensis associated with programmed cell death of parasites and an increase in NO [37].

The aim of this study was to evaluate the in vitro activity of UA on promastigotes and intracellular amastigotes of different Leishmania species in order to select an adequate dose for in vivo efficacy studies. Moreover, topical formulations containing UA were developed, and its efficacy was tested in a chronic model of CL. The activity of parenterally administered UA was assessed on both an acute and chronic model of VL.

\section{Results}

\subsection{Assessment of Topical Formulations and Quantification of $U A$}

UA is a triterpenoid with negligible aqueous solubility. For this reason, it was compounded in semisolid formulations to facilitate its solubility and its topical administration. UA was readily dispersed using a mixture of glycerin: propylene glycol (1:1, w:w) that avoided drug agglomeration after mixing with the preformed emulsion or Orabase in the case of the cream or ointment, respectively. Both formulations exhibited a $\mathrm{pH}$ of 5.5, which is suitable for skin administration. UA was chemically stable over one month at $25 \pm 2{ }^{\circ} \mathrm{C}$. Consistency, color, and $\mathrm{pH}$ were kept unchanged over this period of time. UA concentration was determined using a linear regression calibration curve between 0.78 to $200 \mu \mathrm{g} / \mathrm{mL}\left(\mathrm{y}=3.2423 \mathrm{x}+0.4212 ; \mathrm{R}^{2}=0.9999\right)$.

\subsection{In Vitro Activity against Leishmania spp. and Cytotoxicity against J774 Macrophages}

Miltefosine showed greater in vitro activity on promastigotes than UA. However, UA exhibited an SI ranging from two to four, depending on the Leishmania spp., further in vitro investigations were performed against Leishmania amastigotes (Table 1).

Table 1. In vitro leishmanicidal activity of ursolic acid (UA) on Leishmania promastigotes and cytotoxicity on J774 macrophages. Miltefosine was used as the reference drug.

\begin{tabular}{|c|c|c|c|c|c|c|c|c|c|}
\hline \multirow{2}{*}{ Compound } & \multicolumn{7}{|c|}{$\mathrm{IC}_{50}(\mu \mathrm{g} / \mathrm{mL})^{a}$} & \multicolumn{2}{|r|}{$\mathrm{CC}_{50}(\mu \mathrm{g} / \mathrm{mL})$} \\
\hline & $\begin{array}{l}\text { Leishmania } \\
\text { brazilensis }\end{array}$ & $\mathbf{S I}^{\mathbf{b}}$ & $\begin{array}{l}\text { Leishmania } \\
\text { guyanensis }\end{array}$ & $\mathrm{SI}^{\mathrm{b}}$ & $\begin{array}{l}\text { Leishmania } \\
\text { amazonensis }\end{array}$ & $\mathrm{SI}^{\mathrm{b}}$ & $\begin{array}{l}\text { Leishmania } \\
\text { infantum }\end{array}$ & $\mathbf{S I}^{\mathbf{b}}$ & $\begin{array}{c}\text { J774 } \\
\text { macrophages }\end{array}$ \\
\hline UA & $17.0 \pm 0.92$ & 3.2 & $28.0 \pm 0.09$ & 2 & $14.1 \pm 0.14$ & 4 & $20.9 \pm 1.40$ & 2.7 & $55.8 \pm 3.98$ \\
\hline Miltefosine & $7.16 \pm 0.22$ & 7.9 & $6.85 \pm 0.35$ & 8.2 & $12.44 \pm 0.49$ & 4.6 & $7.19 \pm 0.60$ & 7.9 & $57.1 \pm 4.6$ \\
\hline
\end{tabular}

The anti-leishmanial activity of UA against amastigotes showed a six and three-fold increase compared to extracellular forms of L. amazonensis and L. infantum, respectively (Table 2). Also, the selectivity index of UA was much greater (three and eight-fold higher depending on the strain) than miltefosine. The greater activity of UA observed in amastigotes rather than in promastigotes can be explained by the dual mode of action of this compound not only on the parasite cell but also stimulating the immunological response of the cell host, mainly increasing NO production in macrophages.

Table 2. In vitro leishmanicidal activity of UA on Leishmania intracellular amastigotes. Miltefosine was used as the reference drug.

\begin{tabular}{ccccc}
\hline \multirow{3}{*}{ Compound } & \multicolumn{4}{c}{$\mathrm{IC}_{50}(\mu \mathrm{g} / \mathrm{mL})^{\mathbf{a}}$} \\
\cline { 2 - 5 } & $\begin{array}{c}\text { Leishmania } \\
\text { amazonensis }\end{array}$ & SI $^{\mathbf{b}}$ & $\begin{array}{c}\text { Leishmania }^{\text {infantum }} \\
\text { inf }^{\mathbf{b}}\end{array}$ \\
\hline UA & $2.24 \pm 0.16$ & 24.9 & $6.7 \pm 0.6$ & 8.3 \\
Miltefosine & $20.09 \pm 1.47$ & 2.8 & $23.7 \pm 1.78$ & 2.5 \\
\hline
\end{tabular}

a IC50, concentration of the compound that produced a 50\% reduction in parasites; SD: standard deviation.

${ }^{\mathrm{b}}$ Selectivity index, SI = CC50/IC50. 


\subsection{In Vivo Activity against L. infantum}

\subsubsection{Efficacy in Acute Infection Model of VL in BALB/c}

Supported by the in vitro leishmanicidal efficacy in both intracellular and extracellular forms, UA was also evaluated in vivo in a murine model of acute infection by L. infantum. The compound was administered by the intraperitoneal route at $5 \mathrm{mg} / \mathrm{kg}$ daily for seven consecutive days. The results are summarized in Table 3. UA showed a high parasite growth inhibition corresponding to $99.83 \%$ and $99.78 \%$ reduction in the number of parasites in spleen and liver, respectively, compared to the untreated group $(p<0.05)$. These results suggest a good UA bioavailability from the site of injection to the target organs. No evident signs of toxicity, like loss in weight or hair loss, were observed at the end of the experiment in any of the animals, which suggests that UA was well-tolerated by the infected mice at the administered dose.

Table 3. In vivo antileishmanial efficacy of UA against L. infantum in an acute infection model of visceral leishmaniasis (VL) in BALB/c. Parasite burden of the control (untreated) group and treated group with UA parenterally administered at $5 \mathrm{mg} / \mathrm{kg}$ daily for seven consecutive days. Results are expressed as mean parasite number \pm standard deviation in spleen and liver per milligram.

\begin{tabular}{ccc}
\hline \multirow{2}{*}{ Group } & \multicolumn{2}{c}{$\begin{array}{c}\text { Parasite Burden } \\
\text { (Amastigotes/mg Organ }\left(\times \mathbf{1 0}^{\mathbf{3}}\right)\end{array}$} \\
\cline { 2 - 3 } & Spleen & Liver \\
\hline Treated group (with UA) & $0.18 \pm 0.02 *$ & $0.06 \pm 0.01 *$ \\
Untreated group (control) & $111.84 \pm 2.5$ & $27.96 \pm 7.8$ \\
\hline
\end{tabular}

* Treated control group vs. untreated show significant differences with $p$-value $<0.05$ determined by Mann-Whitney U test.

\subsubsection{Efficacy in Chronic infection Hamster Model of VL}

UA was also evaluated in vivo in a chronic infection model of VL developed in hamsters. Similarly, UA was administered by the intraperitoneal route at $5 \mathrm{mg} / \mathrm{kg}$ daily for seven consecutive days. The results are summarized in Table 4 . In spite of the lack of evident signs of toxicity at the end of the treatment in any of the animals, UA was less effective against chronic than acute infection. At the end of the experiment, the mean weight in the treated group was higher $(145.5 \pm 13.4)$ than in the control group $(126.6 \pm 10.52)$, but there were no statistically significant differences. The natural compound showed a $58 \%$ and $79 \%$ reduction in the number of parasites in spleen and liver, respectively, compared to the untreated group.

Table 4. In vivo anti-leishmanial efficacy of UA against $L$. infantum in chronic infection model of VL in Syrian golden hamsters. Parasite burden of the control (untreated) group and treated group with UA parenterally administered at $5 \mathrm{mg} / \mathrm{kg}$ daily for seven consecutive days is expressed as mean parasite number \pm standard deviation in spleen and liver per milligram.

\begin{tabular}{ccc}
\hline \multirow{2}{*}{ Group } & \multicolumn{2}{c}{$\begin{array}{c}\text { Parasite Burden } \\
\left(\text { Amastigotes/mg Organ }\left(\times \mathbf{1 0}^{\mathbf{6}}\right)\right.\end{array}$} \\
\cline { 2 - 3 } & Spleen & Liver \\
\hline Treated group (with UA) & $9.75 \pm 4.73$ & $4.0 \pm 2.88+^{*}$ \\
Untreated group (control) & $16.78 \pm 5.30$ & $19.04 \pm 7.60$ \\
\hline
\end{tabular}

Treated control group vs. untreated show significant differences with $p$-value $<0.05$ determined by the Mann-Whitney U test.

\subsubsection{In Vivo Activity against L. amazonensis in an Experiment Model of CL}

Prior to topical treatment, disease progression was similar in all animals, and no statistically significant differences were observed after 35 days post-infection (Figure 1). Daily topical administration 
of UA ointment $(0.2 \%)$ for 28 days resulted in a significant $(p<0.05) 42 \%$ and $50.3 \%$ reduction in the lesion size compared to the untreated group at weeks 10 and 15 . However, parasites were not completely eradicated after UA topical administration as inflammation increased at week 15.

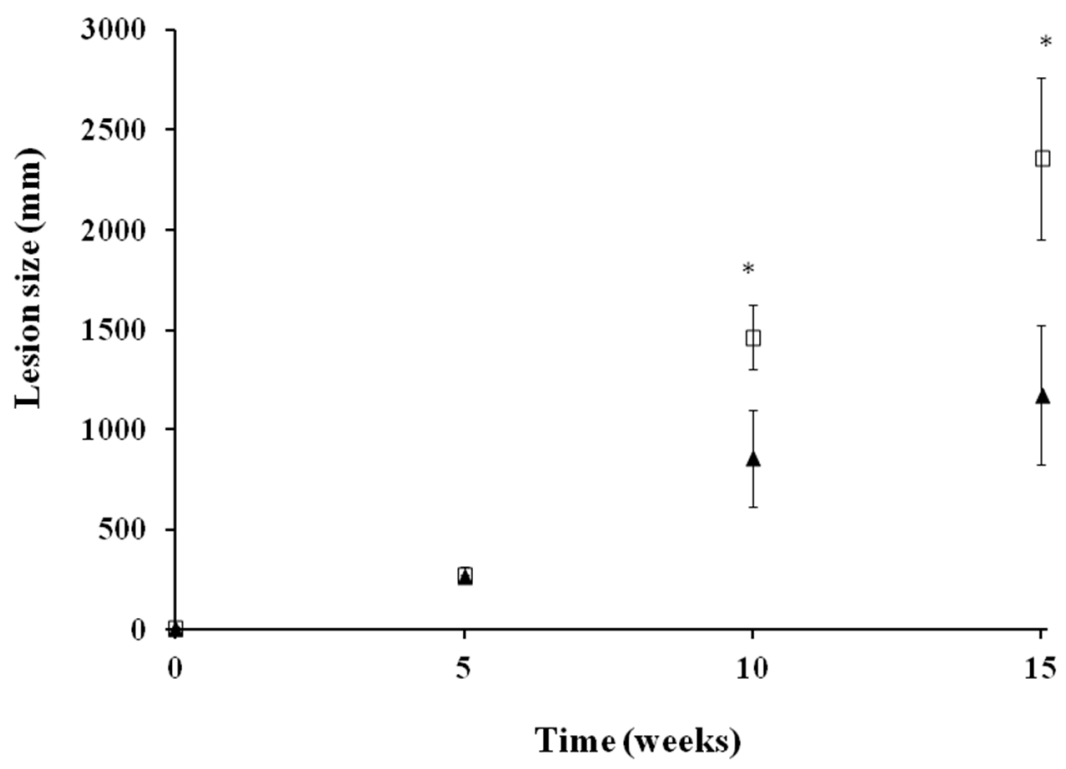

Figure 1. In vivo antileishmanial efficacy of UA ointment against L. amazonensis in a chronic infection model of cutaneous leishmaniasis (CL) in Syrian hamsters. Disease progression was monitored at week $0,5,10$, and 15 . After 35 days post-infection, UA ointment $(0.2 \%)$ was administered once daily for 28 consecutive days in one of the groups. The other group received no treatment. Disease progression was monitored since the treatment was instaured at weeks $0,5,10$, and 15 . Lesion size (mean $\pm \mathrm{SD}$ ) is expressed as the difference between the diameter $(\mathrm{mm})$ of the infected left hind paw and the non-infected right hind paw from each animal. Statistically significant differences $(p<0.05)$ are expressed as: * (U-Mann-Whitney). Key: UA ointment at 0.2\% (-ム-); untreated group (-口-).

In a subsequent study, a higher dose of UA (0.5\%) was tested (Figure 2). Daily topical administration of either UA ointment $(0.5 \%)$ or UA cream $(0.5 \%)$ for 28 days did not result in a significant reduction in the lesion size compared to the untreated group at week 10, although a significant reduction (47.54\%) in inflammation size was achieved at week 15 after the administration of UA ointment. 


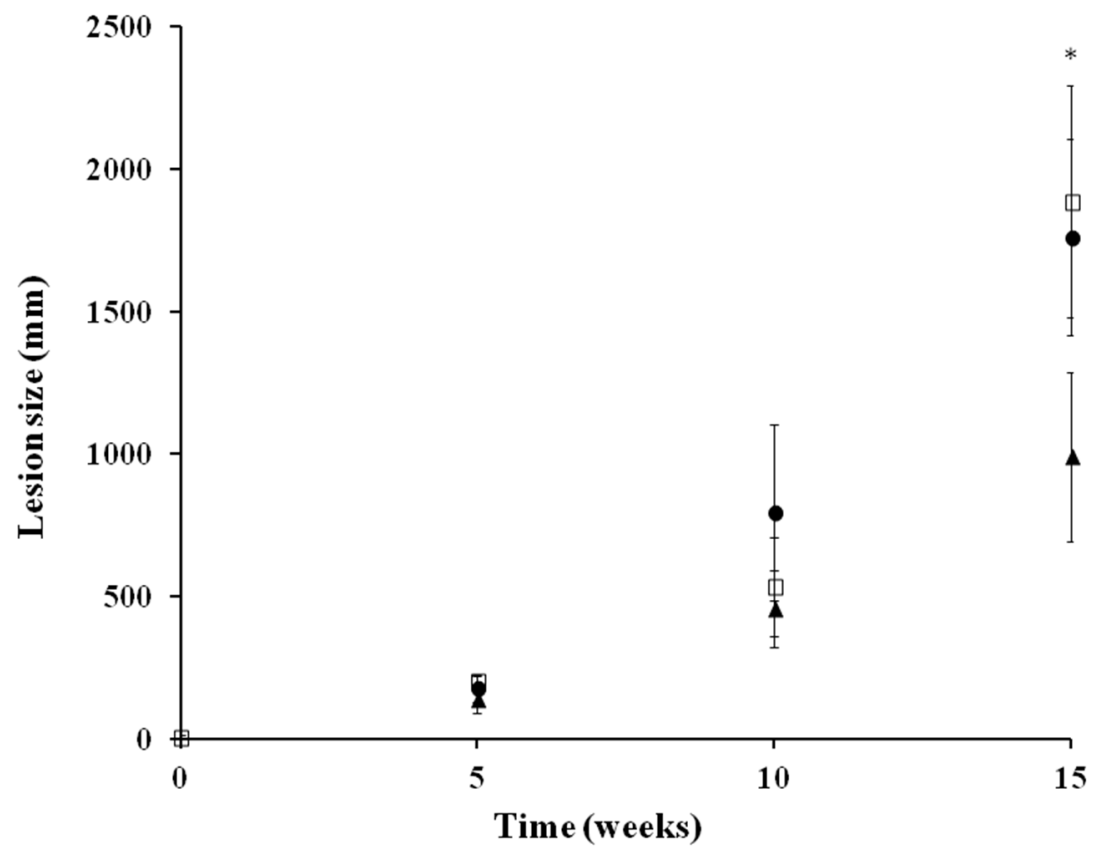

Figure 2. In vivo antileishmanial efficacy of UA ointment and cream against L. amazonensis in a chronic infection model of CL in hamsters. After 35 days post-infection, treatment with UA was topically administered once daily for 28 consecutive days. The first group received UA ointment $(0.5 \%)$. The second group received UA cream $(0.5 \%)$, and the third group was left untreated. Lesion size (mean $\pm \mathrm{SD}$ ) is expressed as the difference between the diameter $(\mathrm{mm})$ of the infected left hind paw and the non-infected right hind paw from each animal. Statistically significant differences $(p<0.05)$ are

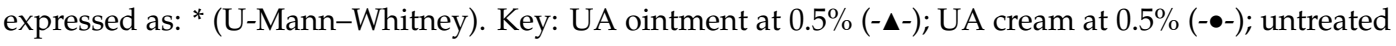
group (-口-).

\subsection{Production of Cytokines}

The efficacy of UA was higher on the intracellular amastigotes than in the promastigotes. This can be linked with an immunological reaction of the macrophages. For this reason, the effect of UA on the production of cytokines in different types of cell cultures was evaluated: (i) naïve splenocytes from mouse spleen and (ii) infected macrophages with L. infantum and L. amazonensis. On the splenocytes, out of the 17 studied cytokines, the following ones showed a significant increase in the presence of UA: GM-CSF, IFN- $\gamma$, IL-4, IL-6, IL-9, IL-10, and decrease of RANTES (Table 5). Nevertheless, IL4, IL6, and IL-10 decreased at higher concentrations of UA. In contrast, higher UA concentrations led to an increase in IFN- $\gamma$, which can make the eradication of intracellular forms of Leishmania favorable, opposite to the decrease in IL-10.

Table 5. Quantification of cytokine production of splenocytes untreated and treated with UA tested at the $\mathrm{IC}_{50}$ concentration corresponding to L. amazonensis and L. infantum.

\begin{tabular}{|c|c|c|c|}
\hline \multirow{2}{*}{$\begin{array}{l}\text { Cytokines } \\
\text { (pg/mL) }\end{array}$} & \multicolumn{3}{|c|}{ Splenocytes Mice BALBc } \\
\hline & Untreated Group & Ursolic Acid $(2.24 \mu \mathrm{g} / \mathrm{mL})$ & Ursolic Acid $(6.7 \mu \mathrm{g} / \mathrm{mL})$ \\
\hline GM-CSF & $11.6 \pm(3)$ & $60.0 \pm(10) *$ & $100.7 \pm(20) *$ \\
\hline IFN- $\gamma$ & 0 & $44.4 \pm(4.2) *$ & $223.4 \pm(87.7)^{* *}$ \\
\hline IL-4 & $3.5 \pm(0.9)$ & $9.3 \pm(1.4)^{*}$ & $6.4 \pm(1.1)$ \\
\hline IL-6 & $42.8 \pm(5.6)$ & $103.1 \pm(11) *$ & $50.2 \pm(7.9)$ \\
\hline IL-9 & $153.1 \pm(20.5)$ & $216.3 \pm(9.6)$ & $232.0 \pm(25.5) *$ \\
\hline IL-10 & $72.2 \pm(17.6)$ & $234.1 \pm(36.2) *$ & $135.8 \pm(33.3) *$ \\
\hline RANTES & $16.2 \pm(0.9)$ & $1.6 \pm(0.3)^{* *}$ & $0 * *$ \\
\hline
\end{tabular}

Statistically significant differences are expressed as ${ }^{*} p$-value $<0.05$ and ${ }^{* *} p$-value $<0.01$ (Tukey's HSD-test). 
It is also key to investigate the production of cytokines on infected macrophages and not only just on lymphocytes. The exposure of Leishmania-infected macrophages to UA led to a significant different production in the cytokines levels depending on the Leishmania strain causing the infection. Except for the production of TNF- $\alpha$ that was exacerbated in both cases, several differences were observed in the levels of: GM-CSF, IFN- $\gamma$, IL-1b, IL-2, IL-6, IL-10, and MCP-1.

In L. infantum-infected macrophages, the production of GM-CSF, IL-6, and IL-10 was augmented compared to the untreated control while the levels of IFN- $\gamma, \mathrm{MCP}-1$, and IL-1b were diminished significantly $(p<0.05)$ when L. amazonensis infected macrophages were exposed to UA (Table 6).

Table 6. Quantification of cytokine production in Leishmania-infected macrophages untreated and treated with UA tested at $\mathrm{IC}_{50}$ values, respectively, for each parasite strain.

\begin{tabular}{ccccc}
\hline \multirow{2}{*}{$\begin{array}{c}\text { Cytokines } \\
(\mathbf{p g} / \mathbf{m L})\end{array}$} & $\begin{array}{c}\text { L. infantum/macrophages } \\
\text { Untreated Mean } \\
\text { (ESM) }\end{array}$ & $\begin{array}{c}\text { Ursolic Acid Mean } \\
\text { (ESM) }\end{array}$ & $\begin{array}{c}\text { Lntreated Mean } \\
\text { (ESM) }\end{array}$ & $\begin{array}{c}\text { Ursolic Acid Mean } \\
\text { (ESM) }\end{array}$ \\
\hline GM-CSF & $23.2 \pm(4.4)$ & $67.3 \pm(2.8)^{*}$ & $49.8 \pm(6.9)$ & $44.8 \pm(0.9)$ \\
IFN- $\gamma$ & $90.2 \pm(125.9)$ & $86.9 \pm(9.8)$ & $139.2 \pm(20.2)$ & $43.5 \pm(26.8)^{*}$ \\
IL-1b & $23.4 \pm(8.5)$ & $24.5 \pm(4.9)$ & $42.3 \pm(5.8)$ & $20 \pm(1.3)^{*}$ \\
IL-2 & 0 & $3.9 \pm(1.1)^{*}$ & $3 \pm(0.4)$ & $1.4 \pm(0.4)^{*}$ \\
IL-6 & $53.4 \pm(11.4)$ & $97.8 \pm(7.2)^{*}$ & $71 \pm(9.5)$ & $66.8 \pm(8.5)$ \\
TNF- $\alpha$ & 0 & $22.72 \pm(3.9)^{*}$ & 0 & $40.22 \pm(6.9)^{*}$ \\
IL-10 & $146.4 \pm(10.8)$ & $258.9 \pm(14.6)^{*}$ & $197.1 \pm(7.9)$ & $184.1 \pm(37.6)$ \\
MCP-1 & $33 \pm(4.0)$ & $50.3 \pm(16.4)$ & $147.9 \pm(19.5)$ & $37.1 \pm(2.2)^{*}$ \\
\hline
\end{tabular}

ESM: Error Standard Mean; Statistically significant differences ( $p$-value $<0.05)$ are expressed as: * (Tukey's HSD-test).

\section{Discussion}

In vitro antileishmanial activity of UA has been previously reported by other authors [35-37]. However, this is one of the first works that reports the in vivo leishmanicidal activity of UA against both VL and CL in different states of the disease. There is a wide variability in the reported in vitro efficacy against Leishmania spp. Many factors can contribute to this variability, but the natural source from which UA is extracted plays a key role in the final activity of this metabolite.

Tan et al. extracted UA from Salvia cilicica roots, which exhibited a very high in vitro activity in the nanomolar range against $L$. donovani and L. major promastigotes ( $\mathrm{IC}_{50}$ equal to 91 and $51.3 \mathrm{nM}$, respectively) and amastigotes $\left(\mathrm{IC}_{50}\right.$ equal to 12.7 and $7.0 \mathrm{nM}$ ) [38]. Moulisha et al. obtained UA from Terminalia arjuna displaying an $\mathrm{IC}_{50}$ of $3.51 \mu \mathrm{g} / \mathrm{mL}$ against L. donovani promastigotes [39]. Odonne et al. extracted UA from Pseudelephantopus spicatus (peruvian plant), which exhibited a high in vitro activity ( $\mathrm{IC}_{50}$ equal to $0.20 \mu \mathrm{M}$ ) against L. amazonensis [40].

On the contrary, UA extracted from Miconialang sdorffii exhibited a very low antileishmanial activity against $L$. amazonensis promastigotes $\left(\mathrm{IC}_{50}\right.$ de $\left.350 \mu \mathrm{M}\right)$ [41]. Chemically synthesized UA showed an $\mathrm{IC}_{50}$ of 20 and $>25 \mu \mathrm{M}$ against L. amazonensis and L. infantum promastigotes, respectively [30].

This variable efficacy is one of the major problems of natural compounds. Extraction and purification processes should also be standardized in order to obtain a compound with reproducible characteristics, making its further use in clinical studies easier. For this reason, chemical synthesized UA (purchased by Sigma-Aldrich) was used to perform the in vitro and in vivo assays.

The higher leishmanicidal activity of UA against intracellular compared to extracellular forms has also been reported by other authors [39]. This fact may be related to its ability to modulate the macrophage nitric oxide production, which would enhance the death of the intracellular parasites [42].

Parenteral administration of UA at $5 \mathrm{mg} / \mathrm{kg}$ for seven consecutive days has shown to significantly reduce parasite growth in liver and spleen in both acute and chronic infection models of VL. In the acute-infection mouse model, although there is parasite growth in the liver and spleen, the infection is usually resolved by a $\mathrm{Th}_{1}$ dependent granulomatous response after multiple weeks [43]. However, VL developed by hamsters closely resembles human VL. It is characterized by a relentless growth of 
parasites in the spleen, liver, and bone marrow, being more complicated to eradicate and usually ends up killing the animal [43]. It has been reported that UA can improve the Th1 immune response in infected mice, which could explain its potent antileishmanial activity [20]. In this sense, the reduction in parasite burden not only in the acute-infection model but also in the chronic model makes UA a promising drug for VL therapy.

Results obtained after UA topical administration against CL were also encouraging suggesting that: (i) higher doses than $0.5 \%$ topically administered can lead to an altered immune-reaction in the cutaneous infected lesion, (ii) UA ointment formulation has a better permeability across the skin than UA cream, and (iii) a successful therapy against CL could be achieved if prolonged topical treatments using UA ointment $(0.2 \%)$ are applied. Despite the fact that creams combine properties, such as lubrication, occlusion, and hydration, that lead to higher patient compliance and better suitability for cutaneous lesions (being especially useful in intertriginous areas where ointments might not be used because they may cause maceration or folliculitis [44,45]), ointments can be more convenient when an occlusive effect is required to enhance drug permeation [45].

The immune response associated with the protection against a Leishmania infection is attributed to the development of a Th1-type immunity characterized by the production of cytokines, such as IFN- $\gamma$, TNF- $\alpha$, IL-12, and GM-CSF, which can lead to the activation of macrophages and production of nitric oxide (NO).

Passero et al. demonstrated that the production of cytokines in an in vivo model of L. infantum-infected hamsters was characterized by an increase in the IL-10 levels at all the doses tested of UA and a decrease in the IFN- $\gamma$ at higher doses [35]. Our in vitro results correlate with those observed by Passero et al. In the latter study, it was also remarked that even though the parasite load were reduced by $96 \%$, the complete eradication of the parasites was not achieved, which can be related to an immunoregulator effect of the parasite on the macrophages [36].

Other studies have shown that the antileishmanial activity of UA depends on two factors: the parasite strain as well as the interaction with the immune system. The production of IFN- $\gamma$ in infected macrophages seems to be more regulated by the presence of the parasites rather than the concentration of UA. This can explain the lack of efficacy of UA in the chronic models of VL [46].

Other studies have shown that GM-CSF has a beneficial output in the disease progression, especially in CL, because it triggers the activation of macrophages to kill Leishmania [47,48]. GM-CSF can improve healing and scarring of cutaneous lesions caused by CL combined con different treatments, such as glucantime or miltefosine $[48,49]$. In our study, an increase in the GM-CSF levels was observed on lymphocytes independently of the dose of the UA tested. However, this increase was only noticeable in those macrophages infected by L. infantum along with higher levels of TNF- $\alpha$. This can explain the higher activity of UA observed against amastigotes compared to promastigotes.

\section{Materials and Methods}

\subsection{Chemical Reagents}

All chemicals used, unless otherwise stated, were obtained from Sigma-Aldrich (Madrid, Spain) or Panreac S.A. (Barcelona, Spain) and used without further purification.

\subsection{Preparation of UA Topical Formulations}

UA cream consisted of an aqueous phase with tween $80(6 \%)$ dispersed in water and an oil phase constituted by white wax $(1 \%)$ and cetyl alcohol $(15 \%)$ melted at $60^{\circ}$. The aqueous phase was heated at $60^{0}$ and then was added onto the oil phase at the same temperature. Continuous stirring was required until the cream reached the desired consistency at room temperature. Finally, UA $(0.5 \%)$ dispersed in a mixture (1:1) of glycerin:propylene glycol (12\%) was added. 
UA ointment was prepared by dispersing the UA $(0.2$ or $0.5 \%)$ in a mixture $(2: 1)$ of glycerin:propylene glycol (5\%), which, in a second step, was mixed with Orabase ${ }^{\circledR}$ (Fagron, Madrid, Spain), a plasticized hydrocarbon gel composed of gelatin, pectin, and sodium carboxymethyl cellulose.

\subsection{Quantification of $U A$ in the Topical Formulations}

UA concentration was assessed by HPLC. Formulations (50 mg) were weighted and dissolved with methanol $(100 \mathrm{~mL})$. Samples were centrifuged at $3000 \times g$ for $10 \mathrm{~min}$, and supernatant was collected and injected in the HPLC. HPLC was equipped with a Jasco PU-1580 pump, a Jasco AS-2050 Plus autosampler, and a Jasco UV-1575UV-visible detector (Jasco, Madrid, Spain). Integration of the peaks was performed with the program Borwin 1.5 for PC (JMBS Developments, Tokyo, Japan). The samples $(100 \mu \mathrm{L})$ were chromatographed over a ThermoHypersil BDS C18 reverse-phase column (Thermo Fisher Scientific, Madrid, Spain) $(200 \times 4.6 \mathrm{~mm}, 5 \mu \mathrm{m})$ at a flow rate of $1 \mathrm{~mL} / \mathrm{min}$. Elution was carried out isocratically with a mobile phase that consisted of a methanol:water $(95: 5, v / v)$ mixture filtered through a $0.45 \mu \mathrm{m}$ hydrophilic polypropylene filter membrane (GH polypro, Pall Corp., DeLand, FL, USA). UA was detected at a wavelength of $210 \mathrm{~nm}$, and its retention time was $5 \mathrm{~min}$.

\subsection{Parasites and Culture Procedure}

The following species of Leishmania were used: an autochthonous isolate of L. infantum (MCAN/ES/96/BCN150) kindly given by Prof. José M. Requena (Universidad Autónoma de Madrid, Madrid, Spain); L. braziliensis 2903, L. amazonensis (MHOM/Br/79/Maria), and L. guyanensis 141/93 were kindly provided by Prof. Alfredo Toraño (Instituto de Salud Carlos III, Madrid, Spain). Promastigotes were cultured in Schneider's Insect Medium supplemented with 10\% heat-inactivated Foetal Bovine Serum (FBS) and $1000 \mathrm{U} / \mathrm{L}$ of penicillin plus $100 \mathrm{mg} / \mathrm{L}$ of streptomycin in $25 \mathrm{~mL}$ culture flasks at $26^{\circ} \mathrm{C}$.

\subsubsection{In Vitro Promastigote Susceptibility Assay}

The assay was performed as previously described [50]. Briefly, log-phase promastigotes $\left(2.5 \times 10^{5}\right.$ parasites/well) were cultured in 96-well plastic plates. Compounds (UA and miltefosine) were dissolved in dimethylsulfoxide (DMSO) and different concentrations of each (100, 50, 25, 12.5, 6.25 3.12, 1.56, and $0.78 \mu \mathrm{g} / \mathrm{mL}$ ) were carried out up to $200 \mu \mathrm{L}$ final volume. Growth control and signal-to-noise were also included. The final solvent (DMSO) concentrations never exceeded $0.5 \%(v / v)$ warranting no effect on parasite proliferation or morphology. After $48 \mathrm{~h}$ at $26^{\circ} \mathrm{C}, 20 \mu \mathrm{L}$ of a $2.5 \mathrm{mM}$ resazurin (Sigma-Aldrich, Madrid, Spain) solution was added to each well, and the plates were returned to the incubator for another $3 \mathrm{~h}$. The Relative Fluorescence Units (RFU) (535-590 nm excitation-emission wavelength) was determined in a fluorometer (Infinite 200Tecan i-Control, Barcelona, Spain). Growth inhibition (GI) was calculated by the following equation:

$$
G I(\%)=100-\frac{R F U \text { treated wells }- \text { RFU signal to noise }}{\text { RFU untreated wells }- \text { RFU signal to noise }} \times 100
$$

All tests were carried out in triplicate. Miltefosine was used as the reference drug and was evaluated under the same conditions. The efficacy of each compound was estimated by calculating the $\mathrm{IC}_{50}$ (concentration of the compound that produced a $50 \%$ reduction in parasites) using a multinomial probit analysis incorporated in SPSS software v21.0 (IBM, Madrid, Spain).

\subsubsection{In Vitro Intracellular Amastigote Susceptibility Assay}

The assay was carried out as previously described [51]. Briefly, $5 \times 10^{4} \mathrm{J774}$ macrophages and stationary promastigotes in a 1:10 ratio were seeded in each well of a microtiter plate, suspended in $200 \mu \mathrm{L}$ of culture medium and incubated for $24 \mathrm{~h}$ at $33{ }^{\circ} \mathrm{C}$ in $5 \% \mathrm{CO}_{2}$ chamber. After this first incubation, the temperature was increased up to $37^{\circ} \mathrm{C}$ for another $24 \mathrm{~h}$. Thereafter, cells were washed several times in culture medium by centrifugation at $1500 \mathrm{rpm}$ for $5 \mathrm{~min}$ in order to remove free 
non-internalized promastigotes. Finally, the supernatant was replaced by $200 \mu \mathrm{L} /$ well of culture medium containing 2-fold serial dilutions of the test compounds (50, 25, 12.5, 6.25 3.12, 1.56, 0.78, and $0.39 \mu \mathrm{g} / \mathrm{mL}$ ). Growth control and signal-to-noise were also included. All tests were carried out in a triplicate assay. Following incubation for $48 \mathrm{~h}$ at $37^{\circ} \mathrm{C}, 5 \% \mathrm{CO}_{2}$, the culture medium was replaced by $200 \mu \mathrm{L} /$ well of the lysis solution (RPMI-1640 with $0.048 \%$ HEPES and $0.006 \%$ SDS) and incubated at room temperature for $20 \mathrm{~min}$. Thereafter, the plates were centrifuged at $3500 \mathrm{rpm}$ for $5 \mathrm{~min}$, and the lysis solution was replaced by $200 \mu \mathrm{L} /$ well of Schneider's insect medium. The culture plates were then incubated at $26{ }^{\circ} \mathrm{C}$ for another 4 days to allow the transformation of viable amastigotes into promastigotes and proliferation. Afterward, $20 \mu \mathrm{L} /$ well of $2.5 \mathrm{mM}$ resazurin was added and incubated for another $3 \mathrm{~h}$. Finally, fluorescence emission was measured, and $\mathrm{IC}_{50}$ was estimated as described above. All tests were carried out in triplicate. Miltefosine was used as the reference drug and was evaluated at the same conditions.

\subsubsection{Cytotoxicity Assay on Macrophages}

The assay was carried out as previously described [52]. J774 macrophages cell lines were seeded $\left(5 \times 10^{4}\right.$ cells/well $)$ in 96-well flat-bottom microplates with $100 \mu \mathrm{L}$ of RPMI 1640 medium. The cells were allowed to attach for $24 \mathrm{~h}$ at $37^{\circ} \mathrm{C}, 5 \% \mathrm{CO}_{2}$, and the medium was replaced by different concentrations of the compounds in $200 \mu \mathrm{L}$ of medium and exposed for another $24 \mathrm{~h}$. Growth controls and signal-to-noise were also included. Afterward, a volume of $20 \mu \mathrm{L}$ of the $2.5 \mathrm{mM}$ resazurin solution was added, and plates were returned to the incubator for another $3 \mathrm{~h}$ to evaluate cell viability. The reduction of resazurin was determined by fluorometry as in the promastigote assay. Each concentration was assayed three times. Cytotoxicity effect of compounds was defined as the $50 \%$ reduction of cell viability of treated culture cells with respect to untreated culture $\left(\mathrm{CC}_{50}\right)$.

\subsection{In Vivo Studies}

Male BALB/c mice of 20-25 g body weight and male golden hamsters (Mesocricetus auratus) of 60-70 g were obtained from Harlan Interfauna Ibérica S.A. (Barcelona, Spain). Animals were allocated in plastic cages in a $12 \mathrm{~h}$ dark-light cycle animal with controlled temperature $\left(25^{\circ} \mathrm{C}\right)$ and humidity $(70 \%)$. Water and food were unrestricted throughout the study. All the animals were handled according to the European Union legislation Directive 2010/63/EU and Spanish law Real Decreto 53/2013 on the protection of animals used for scientific purposes with an ethical committee code CEXAN170415.

\subsubsection{Acute Infection Model of VL in BALB/c}

Each mouse was infected by an intracardiac route with $1 \times 10^{7}$ promastigotes of L. infantum according to a model previously described by Dea-Ayuela et al. [53]. Mice were randomly split into two groups each of six animals. Treatment started on day thirty-five post-infection and lasted for seven consecutive days. One group received UA at $5 \mathrm{mg} / \mathrm{kg}$ once daily intraperitoneally administered in $0.1 \mathrm{~mL}$ of saline solution. The other group was left untreated (control group). One week after the last treatment, all mice were sacrificed, and parasite burden in liver and spleen was estimated by the limit dilution assay describe elsewhere [54]. Briefly, spleen $(0.15 \mathrm{~g})$ and liver $(0.5 \mathrm{~g})$ were homogenized in a $5 \mathrm{~mL}$ of PBS- $50 \mathrm{mM}$ glucose- $2 \mathrm{mM}$ ethylene diamine tetraacetic acid (EDTA) solution at $4{ }^{\circ} \mathrm{C}$ using a sterilized steel stainless tissue grinder. Cell debris was removed by passage through a glass wool column. The suspension obtained was centrifuged at $1500 \mathrm{rpm}$ for $15 \mathrm{~min}$ at $4{ }^{\circ} \mathrm{C}$. Afterward, the supernatants were discarded, and the pellets were collected and resuspended in Schneider medium supplemented as described above, and then, $200 \mu \mathrm{L}$ of this suspension were transferred to the first well of a 96-well microtiter plate containing medium supplemented with antibiotics, as described above. Serial dilutions were repeated, transferring $100 \mu \mathrm{L}$ from the previous well to the next one and adding $100 \mu \mathrm{L}$ of Schneider medium. After incubation at $26^{\circ} \mathrm{C}$ for 7 days, microplates were examined using an inverted microscope (Olympus, model IM) at a magnification of 40X, and the presence or absence 
of promastigotes in each well was recorded. The final titer was the last dilution for which the well contained at least one parasite [54].

\subsubsection{Chronic Infection Model of VL in Golden Hamster}

Chronic infection in Golden hamster was developed, as previously described by Dea-Ayuela et al. [55]. Each hamster was infected by an intracardiac route with $1 \times 10^{7}$ promastigotes of L. infantum. Hamsters were randomly split into two groups of six animals. Treatment started on day thirty-five post-infection and lasted for seven consecutive days. One group received UA at $5 \mathrm{mg} / \mathrm{kg}$ once daily intraperitoneally administered in $0.1 \mathrm{~mL}$ of saline solution. The other group was left untreated (control group). Parasite burden in liver and spleen was estimated by the limit dilution assay described above [54].

\subsubsection{Chronic Infection Model of CL in Golden Hamster}

This experiment was carried out as described by Morais-Teixeira et al., with some modifications [56]. Hamsters were randomly split into two groups of six animals. A suspension $(25 \mu \mathrm{L})$ containing $1 \times 10^{7}$ stationary promastigotes of L. amazonensis in Schneider medium was injected subcutaneously in the footpad of the left hind paw at day 0 . Right hind paw was used as a negative control (no infection). After 5 weeks post-infection, chronic CL was developed, and topical treatment with UA started. In a first experiment, UA ointment $(0.2 \%)$ was administered once daily for 4 consecutive weeks. The other group received no treatment. Disease progression was monitored at weeks $0,5,10$, and 15 using a Vernier caliper to measure footpad size. In a second experiment, hamsters were randomly split into three groups of six animals. One group was treated with UA ointment $(0.5 \%)$, and the other group was tread with UA cream $(0.5 \%)$. As in the first experiment, the third group received no treatment (control group).

\subsection{Effect of $U A$ in the Cytokines Production in Macrophages Infected with Leishmania In Vitro}

In vitro Leishmania infection was carried out as described previously. After the incubation with $200 \mu \mathrm{L} /$ well of culture medium containing $\mathrm{UA}$ at the $\mathrm{IC}_{50}$ concentration previously determined for each of the species (L. infantum and L. amazonensis) for $48 \mathrm{~h}$ the supernatants were collected and stored at $-80{ }^{\circ} \mathrm{C}$ for cytokine quantification.

\subsubsection{Splenocytes Culture and Evaluation of Cytokines Production}

Cell suspension of splenocytes was prepared from the spleen of non-infected BALB/c mice and then was rinsed with RPMI 1640 medium three times. Then, $5 \mathrm{~mL}$ of the cell suspension was added to $1 \mathrm{~mL}$ of ammonium chloride $0.8 \%$ to isolate the splenocytes, and the suspension was again washed three times with RPMI 1640 medium. The splenocytes, $2.5 \times 10^{9}$ cells/L, were cultured in RPMI 1640 medium containing $10 \%$ fetal bovine serum and incubated at $37^{\circ} \mathrm{C}$ for $24 \mathrm{~h}$. After incubation for $24 \mathrm{~h}$, the supernatants were collected and stored at $-80^{\circ} \mathrm{C}$ for cytokine quantification.

\subsubsection{Analysis of Cytokines}

The analyses were carried out by using the Quantibody ${ }^{\circledR}$ array (RayBiotech, Madrid, Spain), a multiplexed sandwich ELISA-based quantitative array platform [57]. Nineteen different markers were analyzed: GM-CSF, IFN $\gamma$, IL-1a, IL-1 $\beta$, IL-2, IL-3, IL-4, IL-5, IL-6, IL-9, IL-10, IL-12, IL-13, IL-17, KC, MCP-1, M-CSF, RANTES, VEGF. A pair of cytokine specific antibodies was used for detection. A capture antibody was first bound to the glass surface. After incubation with the sample, the target cytokine was trapped on the solid surface. A second biotin-labeled detection antibody was added, which recognized a different epitope of the target cytokine. The cytokine-antibody-biotin complex was visualized through the addition of the streptavidin-conjugated $\mathrm{Cy} 3$ equivalent dye, using a laser scanner (GenePix ${ }^{\circledR}$ 4000B Scanner, UCM Facilities, Madrid, Spain). 
A sandwich ELISA kit was used to determine the concentrations of TNF- $\alpha$ according the manufacturer's instructions. Briefly, standards and samples $(50 \mu \mathrm{L})$ were diluted with $50 \mu \mathrm{L}$ of commercial diluting solution in triplicate and incubated for $2 \mathrm{~h}$ at $37^{\circ} \mathrm{C}$. Plates were washed five times, and $100 \mu \mathrm{L}$ of conjugated anti-TNF- $\alpha$ was added and incubated for $1 \mathrm{~h}$ at $37^{\circ} \mathrm{C}$. Finally, $100 \mu \mathrm{L}$ of TMB was added as substrate and incubated for $30 \mathrm{~min}$ in dark at room temperature. The reaction was stopped with $3 \mathrm{M} \mathrm{H}_{2} \mathrm{SO}_{4}$, and the optical densities (OD) were read at $450 \mathrm{~nm}$.

\subsection{Statistics}

\subsubsection{Statistical and Data Analysis for In Vitro Assays}

The efficacy against the parasite $\left(\mathrm{IC}_{50}\right)$ and cytotoxicity effect $\left(\mathrm{CC}_{50}\right)$ of compounds were calculated from Probit analysis using SPSS v21.0 software (IBM, Madrid, Spain) Finally, selective index (SI) was calculated defined as the ratio between $\mathrm{CC}_{50} / \mathrm{IC}_{50}$.

The analysis of cytokines in vitro was performed by Tukey's HSD test using the statistical program IBM $^{\circledR}$ SPSS v21.0 (IBM, Madrid, Spain).

\subsubsection{Statistical and Data Analysis for in Vivo Assays}

The group mean, standard deviation, standard error, and the differences were compared by the Mann-Whitney U nonparametric test, using the statistical programs of Microsoft Excel 2011 ${ }^{\circledR}$ (Microsoft, Washington, DC, USA) and IBM ${ }^{\circledR}$ SPSS v21.0. Statistical significance was set at a $p$-value $<0.05$.

\section{Conclusions}

Currently, plant-based therapies have shown great potential in the treatment of leishmaniasis. However, it is challenging to find a compound that possesses efficacy not only against cutaneous but also against visceral leishmaniasis. In this work, we have demonstrated that UA, a ubiquitous triterpenoid in nature, has a potent antileishmanial efficacy against both VL and CL, having a direct effect on the immunological response of the host. UA parenterally administered at $5 \mathrm{mg} / \mathrm{kg}$ significantly reduced parasite growth in liver and spleen not only in acute-infection but also in a chronic infection model. In addition, UA ointment $(0.2 \%)$ topically administered could constitute a promising therapy against CL being able to penetrate across the skin and to diminish disease progression. In conclusion, formulating UA with suitable excipient can lead to promising treatments for cutaneous and visceral leishmaniasis.

Author Contributions: Conceptualization, M.A.D.-A. and F.B.-F.; methodology, P.B.-R., D.R.S., H.K.R.S. and M.A.D.-A.; validation, P.B.-R., D.R.S. and H.K.R.S.; formal analysis, P.B.-R., D.R.S. and M.A.D.-A.; investigation P.B.-R., D.R.S. and M.A.D.-A.; writing—original draft preparation P.B.-R., D.R.S. and M.A.D.-A.; writing-review and editing, D.R.S. and M.A.D.-A.; resources, F.B.-F., J.J.T., M.A.D.-A.; supervision, F.B.-F. and J.J.T.; project administration, M.A.D.-A.; funding acquisition, M.A.D.-A. and F.B.-F. All authors have read and agreed to the published version of the manuscript.

Funding: This work was supported by the University CEU-Cardenal Herrera (Project PRCEU-UCH09-19) and Spanish Agency for International Cooperation and Development (AECID) A-024457/09 and AP/038991/11. UCM-Cooperation Program 14-15. P. Bilbao-Ramos was supported by a Research Fellowship grant from the MAEC-AECID.

Conflicts of Interest: The authors declare no conflict of interest.

\section{References}

1. Alvar, J.; Vélez, I.D.; Bern, C.; Herrero, M.; Desjeux, P.; Cano, J.; Jannin, J.; den Boer, M.; WHO Leishmaniasis Control Team. Leishmaniasis worldwide and global estimates of its incidence. PLOS ONE 2012, 7, e35671. [CrossRef] [PubMed]

2. WHO. Leishmaniasis. 2015. Available online: http://www.who.int/leishmaniasis/en/ (accessed on 15 May 2018).

3. Yarbuh, A.L.; Premoli-de-Percoco, G.; Valera, M. Localized cutaneous leishmaniasis using polymerase chain reaction: A venezuelan family report. Parasitol. Dia 1997, 21, 3-4. 
4. Grimaldi, G., Jr.; Tesh, R.B. Leishmaniases of the New World: Current concepts and implications for future research. Clin. Microbiol. Rev. 1993, 6, 230-250. [CrossRef] [PubMed]

5. Alvar, J.; Croft, S.; Olliaro, P. Chemotherapy in the treatment and control of leishmaniasis. Adv. Parasitol. 2006, 61, 223-274.

6. Freitas-Junior, L.H.; Chatelainb, E.; Kima, H.A.; Siqueira-Neto, J. Visceral leishmaniasis treatment: What do we have, what do we need and how to deliver it? Int. J. Parasitol. Drugs Drug Resis. 2012, 2, 11-19. [CrossRef]

7. Frézard, F.; Demicheli, C.; Ribeiro, R.R. Pentavalent antimonials: New perspectives for old drugs. Molecules 2009, 14, 2317-2336. [CrossRef]

8. Roy, P.; Das, S.; Auddy, R.G.; Mukherjee, A. Biological targeting and drug delivery in control of Leishmaniasis. J. Cell Anim. Biol. 2012, 6, 73-87.

9. Sundar, S.; Olliaro, P.L. Miltefosine in the treatment of leishmaniasis: Clinical evidence for informed clinical risk management. Ther. Clin. Risk Manag. 2007, 3, 733-740.

10. Rocha, L.G.; Almeida, J.R.; Macêdo, R.O.; Barbosa-Filho, J.M. A review of natural products with antileishmanial activity. Phytomedicine 2005, 12, 514-535. [CrossRef]

11. Jucá, M.M.; Cysne Filho, F.M.S.; de Almeida, J.C.; Mesquita, D.D.S.; Barriga, J.R.M.; Dias, K.C.F.; Barbosa, T.M.; Vasconcelos, L.C.; Leal, L.K.A.M.; Ribeiro, J.E.; et al. Flavonoids: Biological activities and therapeutic potential. Nat. Prod. Res. 2018, 16,1-14. [CrossRef]

12. Nazemiyeh, H.; Bahadori, F.; Delazar, A.; Ay, M.; Topcu, G.; Kolak, U.; Nahar, L.; Auzie, A.A.; Sarker, S.D. Tricetin 4'-O- $\alpha$-L-rhamnopyranoside: A new flavonoid from the aerial parts of Erica arborea. Chem. Nat. Compd. 2008, 44, 174-177. [CrossRef]

13. Kowalczyk, E.; Krzesiński, P.; Kura, M.; Szmigiel, B.; Błaszczyk, J. Anthocyanins in medicine. Pol. J. Pharmacol. 2003, 55, 699-702. [PubMed]

14. Abebe, W. Herbal medication: Potential for adverse interactions with analgesic drugs. J. Clin. Pharm. Ther. 2002, 27, 391-401. [CrossRef] [PubMed]

15. Bhattacharya, S.; Biswas, M.; Haldar, P.K. The triterpenoid fraction from Trichosanthes dioica root exhibits in vitro antileishmanial effect against Leishmania donovani promastigotes. Pharmacog. Res. 2013, 5, 109-112. [CrossRef]

16. Szakiel, A.; Paczkowski, C.; Pensec, F.; Bertsch, C. Fruit cuticular waxes as a source of biologically active triterpenoids. Phytochem. Rev. 2012, 11, 263-284. [CrossRef]

17. Checker, R.; Sandur, S.K.; Sharma, D.; Patwardhan, R.S.; Jayakumar, S.; Kohli, V.; Sethi, G.; Aggarwal, B.B.; Sainis, K.B. Potent anti-inflammatory activity of ursolic acid, a triterpenoid antioxidant, is mediated through suppression of NF-kappaB, AP-1 and NF-AT. PLoS ONE 2012, 7, e31318. [CrossRef]

18. Torres-Santos, E.C.; Lopes, D.; Oliveira, R.R.; Carauta, J.P.; Falcao, C.A.; Kaplan, M.A.; Rossi-Bergmann, B. Antileishmanial activity of isolated triterpenoids from Pourouma guianensis. Phytomedicine 2004, 11, 114-120. [CrossRef]

19. Innocente, A.M.; Silva, G.N.; Cruz, L.N.; Moraes, M.S.; Nakabashi, M.; Sonnet, P.; Gosmann, G.; Garcia, C.R.; Gnoatto, S.C. Synthesis and antiplasmodial activity of betulinic acid and ursolic acid analogues. Molecules 2012, 17, 12003-12014. [CrossRef]

20. Yamamoto, E.S.; Campos, B.L.; Laurenti, M.D.; Lago, J.H.; Grecco Sdos, S.; Corbett, C.E.; Passero, L.F. Treatment with triterpenic fraction purified from Baccharis uncinella leaves inhibits Leishmania (Leishmania) amazonensis spreading and improves Th1 immune response in infected mice. Parasitol. Res. 2014, 113, 333-339. [CrossRef]

21. da Silva Ferreira, D.; Esperandim, V.R.; Toldo, M.P.; Kuehn, C.C.; do Prado Junior, J.C.; Cunha, W.R.; e Silva, M.L.; de Albuquerque, S. In vivo activity of ursolic and oleanolic acids during the acute phase of Trypanosoma cruzi infection. Exp. Parasitol. 2013, 134, 455-459. [CrossRef]

22. Liu, J. Oleanolic acid and ursolic acid: Research perspectives. J. Ethnopharmacol. 2005, 100, 92-94. [CrossRef] [PubMed]

23. Martin-Cordero, C.; Reyes, M.; Ayuso, M.J.; Toro, M.V. Cytotoxic triterpenoids from Erica andevalensis. Z. Nat. C 2001, 56, 45-48. [CrossRef]

24. Wang, X.H.; Zhou, S.Y.; Qian, Z.Z.; Zhang, H.L.; Qiu, L.H.; Song, Z.; Zhao, J.; Wang, P.; Hao, X.S.; Wang, H.Q. Evaluation of toxicity and single-dose pharmacokinetics of intravenous ursolic acid liposomes in healthy adult volunteers and patients with advanced solid tumors. Expert Opin. Drug Metab. Toxicol. 2013, 9, 117-125. [CrossRef] [PubMed] 
25. Zhu, Z.; Qian, Z.; Yan, Z.; Zhao, C.; Wang, H.; Ying, G. A phase I pharmacokinetic study of ursolic acid nanoliposomes in healthy volunteers and patients with advanced solid tumors. Int. J. Nanomed. 2013, 8, 129-136.

26. do Nascimento, P.G.; Lemos, T.L.; Bizerra, A.M.; Arriaga, A.M.; Ferreira, D.A.; Santiago, G.M.; Braz-Filho, R.; Costa, J.G. Antibacterial and antioxidant activities of ursolic acid and derivatives. Molecules 2013, 19, 1317-1327. [CrossRef]

27. Alqahtani, A.; Hamid, K.; Kam, A.; Wong, K.H.; Abdelhak, Z.; Razmovski-Naumovski, V.; Chan, K.; Li, K.M.; Groundwater, P.W.; Li, G.Q. The pentacyclic triterpenoids in herbal medicines and their pharmacological activities in diabetes and diabetic complications. Curr. Med. Chem. 2013, 20, 908-931.

28. Kashiwada, Y.; Nagao, T.; Hashimoto, A.; Ikeshiro, Y.; Okabe, H.; Cosentino, L.M.; Cosentino, L.M.; Lee, K.H. Anti-AIDS agents 38. Anti-HIV activity of 3-O-acyl ursolic acid derivatives. J. Nat. Prod. 2000, 63, 1619-1622. [CrossRef]

29. Horiuchi, K.; Shiota, S.; Hatano, T.; Yoshida, T.; Kuroda, T.; Tsuchiya, T. Antimicrobial activity of oleanolic acid from Salvia officinalis and related compounds on vancomycin-resistant enterococci (VRE). Biol. Pharm. Bull. 2007, 30, 1147-1149. [CrossRef]

30. Gnoatto, S.C.; Dalla Vechia, L.; Lencina, C.L.; Dassonville-Klimpt, A.; Da Nascimento, S.; Mossalayi, D.; Guillon, J.; Gosmann, G.; Sonnet, P. Synthesis and preliminary evaluation of new ursolic and oleanolic acids derivatives as antileishmanial agents. J. Enzyme Inhib. Med. Chem. 2008, 23, 604-610. [CrossRef]

31. Kwon, T.H.; Lee, B.; Chung, S.H.; Kim, D.H.; Lee, Y.S. Synthesis and NO Production inhibitory activities of ursolic acid and oleanolic acid derivatives. Bull. Korean Chem. Soc. 2009, 30, 119-123. [CrossRef]

32. Zerin, T.; Lee, M.; Jang, W.S.; Nam, K.W.; Song, H.Y. Anti-inflammatory potential of ursolic acid in Mycobacterium tuberculosis-sensitized and concanavalin A-stimulated cells. Mol. Med. Rep. 2016, 13, 2736-2744. [CrossRef] [PubMed]

33. Lopez-Garcia, S.; Castaneda-Sanchez, J.I.; Jimenez-Arellanes, A.; Dominguez-Lopez, L.; Castro-Mussot, M.E.; Hernandez-Sanchez, J.; Luna-Herrera, J. Macrophage Activation by Ursolic and Oleanolic Acids during Mycobacterial Infection. Molecules 2015, 20, 14348-14364. [CrossRef] [PubMed]

34. Choi, W.H.; Lee, I.A. The Mechanism of Action of Ursolic Acid as a Potential Anti-Toxoplasmosis Agent, and Its Immunomodulatory Effects. Pathogens 2019, 8, 61. [CrossRef] [PubMed]

35. Passero, L.F.; Bonfim-Melo, A.; Corbett, C.E.; Laurenti, M.D.; Toyama, M.H.; de Toyama, D.O.; Romoff, P.; Fávero, O.A.; dos Grecco, S.S.; Zalewsky, C.A.; et al. Anti-leishmanial effects of purified compounds from aerial parts of Baccharis uncinella C. DC. (Asteraceae). Parasitol. Res. 2011, 108, 529-536. [CrossRef] [PubMed]

36. Jesus, J.A.; Fragoso, T.N.; Yamamoto, E.S.; Laurenti, M.D.; Silva, M.S.; Ferreira, A.F.; Lago, J.H.; Santos-Gomes, G.; Passero, L.F. Therapeutic effect of ursolic acid in experimental visceral leishmaniasis. Int. J. Parasitol. Drugs Drug Resist. 2017, 7, 1-11. [CrossRef] [PubMed]

37. Yamamoto, E.S.; Campos, B.L.; Jesus, J.A.; Laurenti, M.D.; Ribeiro, S.P.; Kallas, E.G.; Rafael-Fernandes, M.; Santos-Gomes, G.; Silva, M.S.; Sessa, D.P.; et al. The Effect of Ursolic Acid on Leishmania (Leishmania) amazonensis is Related to Programed Cell Death and Presents Therapeutic Potential in Experimental Cutaneous Leishmaniasis. PLoS ONE 2015, 10, e0144946. [CrossRef]

38. Tan, N.; Kaloga, M.; Radtke, O.A.; Kiderlen, A.F.; Oksuz, S.; Ulubelen, A.; Kolodziej, H. Abietane diterpenoids and triterpenoic acids from Salvia cilicica and their antileishmanial activities. Phytochemistry 2002, 61, 881-884. [CrossRef]

39. Moulisha, B.; Kumar, G.A.; Kanti, H.P. Anti-leishmanial and anti-cancer activities of a pentacyclic triterpenoid isolated from the leaves of Terminalia arjuna Combretaceae. Trop. J. Pharm. Res. 2010, 9, 135-140. [CrossRef]

40. Odonne, G.; Herbette, G.; Eparvier, V.; Bourdy, G.; Rojas, R.; Sauvain, M.; Stien, D. Antileishmanial sesquiterpene lactones from Pseudelephantopus spicatus, a traditional remedy from the Chayahuita Amerindians (Peru). Part III. J. Ethnopharmacol. 2011, 137, 875-879. [CrossRef]

41. Peixoto, J.A.; Andrade, E.S.M.L.; Crotti, A.E.; Cassio Sola Veneziani, R.; Gimenez, V.M.; Januario, A.H.; Groppo, M.; Magalhães, L.G.; Dos Santos, F.F.; Albuquerque, S.; et al. Antileishmanial activity of the hydroalcoholic extract of Miconia langsdorffii, isolated compounds, and semi-synthetic derivatives. Molecules 2011, 16, 1825-1833. [CrossRef]

42. You, H.J.; Choi, C.Y.; Kim, J.Y.; Park, S.J.; Hahm, K.S.; Jeong, H.G. Ursolic acid enhances nitric oxide and tumor necrosis factor-alpha production via nuclear factor-kappa B activation in the resting macrophages. FEBS Lett. 2001, 509, 156-160. [CrossRef] 
43. Lafuse, W.P.; Story, R.; Mahylis, J.; Gupta, G.; Varikuti, S.; Steinkamp, H.; Oghumu, S.; Satoskar, A.R. Leishmania donovani infection induces anemia in hamsters by differentially altering erythropoiesis in bone marrow and spleen. PLoS ONE 2013, 8, e59509. [CrossRef] [PubMed]

44. Ference, J.D.; Last, A.R. Choosing topical corticosteroids. Am. Fam. Physician 2009, 79, 135-140. [PubMed]

45. Wong, T.W.; Chiu, H.C.; Chang, C.H.; Lin, L.J.; Liu, C.C.; Chen, J.S. Silicone cream occlusive dressing-a novel noninvasive regimen in the treatment of keloid. Dermatology 1996, 192, 329-333. [CrossRef] [PubMed]

46. Jesus, J.A.; Lago, J.H.; Laurenti, M.D.; Yamamoto, E.S.; Passero, L.F. Antimicrobial activity of oleanolic and ursolic acids: An update. Evid. Based Complement. Alternat. Med. 2015, 2015, 620472. [CrossRef]

47. Ho, J.L.; Reed, S.G.; Wick, E.A.; Giordano, M. Granulocyte-macrophage and macrophage colony-stimulating factors activate intramacrophage killing of Leishmania mexicana amazonensis. J. Infect. Dis. 1990, 162, 224-230.

48. Almeida, R.P.; Brito, J.; Machado, P.L.; de Jesus, A.R.; Schriefer, A.; Guimarães, L.H.; Carvalho, E.M. Successful treatment of refractory cutaneous leishmaniasis with GM-CSF and antimonials. Am. J. Trop. Med. Hyg. 2005, 73, 79-81. [CrossRef]

49. Miltefosine and GM-CSF in Cutaneous Leishmaniasis. Available online: https://clinicaltrials.gov/ct2/show/ NCT03023111 (accessed on 15 April 2019).

50. Bilbao-Ramos, P.; Galiana-Rosello, C.; Dea-Ayuela, M.A.; Gonzalez-Alvarez, M.; Vega, C.; Rolon, M.; Pérez-Serrano, J.; Bolás-Fernández, F.; González-Rosende, M.E. Nuclease activity and ultrastructural effects of new sulfonamides with anti-leishmanial and trypanocidal activities. Parasitol. Int. 2012, 61, 604-613. [CrossRef]

51. Bilbao-Ramos, P.; Sifontes-Rodriguez, S.; Dea-Ayuela, M.A.; Bolas-Fernandez, F. A fluorometric method for evaluation of pharmacological activity against intracellular Leishmania amastigotes. J. Microbiol. Methods 2012, 89, 8-11. [CrossRef]

52. Galiana-Rosello, C.; Bilbao-Ramos, P.; Dea-Ayuela, M.A.; Rolon, M.; Vega, C.; Bolas-Fernandez, F.; García-España, E.; Alfonso, J.; Coronel, C.; González-Rosende, M.E. In vitro and in vivo antileishmanial and trypanocidal studies of new N-benzene- and N-naphthalenesulfonamide derivatives. J. Med. Chem. 2013, 56, 8984-8998. [CrossRef]

53. Dea-Ayuela, M.A.; Castillo, E.; Gonzalez-Alvarez, M.; Vega, C.; Rolon, M.; Bolas-Fernandez, F.; Borrás, J.; González-Rosende, M.E. In vivo and in vitro anti-leishmanial activities of 4-nitro-N-pyrimidin- and N-pyrazin-2-ylbenzenesulfonamides, and N2-(4-nitrophenyl)-N1-propylglycinamide. Bioorg. Med. Chem. 2009, 17, 7449-7456. [CrossRef] [PubMed]

54. Titus, R.G.; Marchand, M.; Boon, T.; Louis, J.A. A limiting dilution assay for quantifying Leishmania major in tissues of infected mice. Parasite Immunol. 1985, 7, 545-555. [CrossRef] [PubMed]

55. Dea-Ayuela, M.A.; Rama-Iniguez, S.; Alunda, J.M.; Bolas-Fernandez, F. Setting new immunobiological parameters in the hamster model of visceral leishmaniasis for in vivo testing of antileishmanial compounds. Vet. Res. Commun. 2007, 31, 703-717. [CrossRef] [PubMed]

56. Morais-Teixeira, E.D.; Carvalho, A.S.; Costa, J.C.; Duarte, S.L.; Mendonça, J.S.; Boechat, N.; Rabello, A. In vitro and in vivo activity of meglumine antimoniate produced at Farmanguinhos-Fiocruz, Brazil, against Leishmania (Leishmania) amazonensis, L (L.) chagasi and L (Viannia) braziliensis. Mem. Inst. Oswaldo Cruz 2008, 103, 358-362. [CrossRef] [PubMed]

57. Perez-Cantero, A.; Serrano, D.R.; Navarro-Rodriguez, P.; Schatzlein, A.G.; Uchegbu, I.F.; Torrado, J.J.; Capilla, J. Increased Efficacy of Oral Fixed-Dose Combination of Amphotericin B and AHCC((R)) Natural Adjuvant against Aspergillosis. Pharmaceutics 2019, 11, 456. [CrossRef] [PubMed]

Sample Availability: Samples of the compounds are available from the authors. 